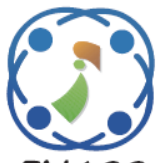

\title{
Overlapped Semantic Age Group Estimation Using Hybrid PCA and Log Gabor Filter
}

\author{
Santhosh Kumar Gangadharaiah ${ }^{1 *}$ Hosahally Narayangowda Suresh ${ }^{2}$ \\ ${ }^{1}$ East West Institute of Technology, Electronics and Communication Engineering, India \\ ${ }^{2}$ Bangalore Institute of Technology, Electronics and Communication Engineering, India \\ * Corresponding author's Email: santhoshphd2015@ gmail.com
}

\begin{abstract}
Automatic extraction of soft biometric characteristics of face image is an emerging research field in recent years. Among these soft biometrics, age estimation is very useful for several applications, like video surveillance, business intelligence, and search optimization in large databases. Generally, facial aging effects perceived in two main forms like, growth related transformations and textural variation. So, in order to generate an effective age classifier, both dimension and texture information should be used together. In this work, an age estimation system that is combination of dimension feature (Hybrid Principal Component Analysis (HPCA)) and textural feature (Log Gabor filter) for feature extraction, is used. The extracted feature values are classified by employing a multi-objective classifier named as Multi-Support Vector Machine (M-SVM). The result of the experiment shows that the proposed approach delivers a better age estimation rate on overlapping age group classes.
\end{abstract}

Keywords: Age estimation, Hybrid principal component analysis, Log Gabor filter, Multi-support vector machine.

\section{Introduction}

Human age estimation based on facial images has become an attractive research area in recent years. Estimating human age by seeing his or her face is very hard not only for the existing computer vision systems, but also for humans in some circumstances [1-2]. Aging pattern of each person is determined by many internal and external factors like genetics, health, lifestyle, and even weather conditions [3]. In order to achieve successful results in applications like age estimation or age classification, the dataset that is used to train the algorithm must contain all these factors [4-5]. Therefore, the collection of training data is another difficulty of research on age estimation [6]. It is really hard to collect face images of the same person at different ages and it is very important to assign each instance to the right age class [7]. In order to have a general and qualified aging pattern that overcomes the negative influences of individual differences, a complete and accurately labeled face aging database is needed [8]. So, we have undertaken East West College (EWC) and
MORPH dataset for age estimation analysis. Regardless of these present difficulties, age estimation can be used in a wide range of smart human-machine applications, for an instant, limiting access to age-appropriate internet or television contents [9].

Facial aging is a problem in face recognition, because simulating the appearance of a person across years may help recognizing his or her face [10-11]. Most of the existing facial age estimation methods usually employ handcrafted feature descriptors like Local Binary Pattern (LBP), color moments, etc., for face representation, which require strong prior knowledge [12-13]. Also, a few attempts like preprocessing is performed on learning-based feature representation in facial age estimation, which learn discriminative features directly from raw pixels. Features learned by these methods are very difficult, because these feature descriptors are not robust enough to local variations [14-15]. To overcome these difficulties, here we have proposed an age estimation system that is combination of dimension feature (Hybrid Principal Component Analysis 
(HPCA)) and textural feature (Log Gabor filter) for feature extraction. This proposed method is classified into three stages: pre-processing, feature extraction and classification. In the first stage, it is essential to detect the face region from input images. After detecting the facial region, normalization is performed for removing the unwanted noise from the facial image. Secondly, the overlapped age group estimation is performed by applying semantic feature extraction using HPCA and Log-Gabor feature descriptors. After obtaining the feature values, the classification of overlapped age groups is done by employing M-SVM classifier.

The rest of this paper is organized as follows: Section 2 survey several existing age estimation methods. In section 3, semantic feature extraction methods are presented with multi objective classification. Section 4 shows comparative experimental results in age estimation for two databases (EWC and MORPH) and Section 5 provides some concluding remarks.

\section{Literature review}

A. Günay, and V.V. Nabiyev [16] presented a new age estimation technique by utilizing hybrid features. In this literature, the author has used models like Active Appearance Model (AAM) and LBP for global and local feature extraction of face image. After the extraction of features the resulting functions were combined with a decision level fusion approach. Finally, the experimental outcome showed that the proposed technique outperforms previous age estimation approaches in terms of mean absolute error. This literature only concentrated on internal factors, not much attention on external attributes. So, the typical attributes will affect the influence rate of the aging pattern.

T. Wu, P. Turaga, and R. Chellappa [17] presented a model for arranging $2 \mathrm{D}$ landmarks on a face with an affine invariant shape representation. This shape representation indicates a grassmann manifold analysis of the shape space. The age estimation was verified by utilizing geometry based manifold. In numerous applications, the accurate verification of age estimation was considered as one of the major issues. An age recognized data were encoded in shape with face texture. Proposed approach was more effective and also flexible for employing standalone classifier or age estimator.

J.K. Pontes, A.S. Britto, C. Fookes, and A.L. Koerich [18] proposed a flexible hierarchical age estimation methodology which contains a multi-class Support Vector Machine (SVM) to categorize a subject into an age group followed by a Support Vector Regression (SVR) to evaluate an exact age and employ global feature descriptors like AAM, Gabor wavelets, and LBP to extract the local features. It shows highly discriminate analysis to produce a very significant result for AE. However, some of the factors affect the measurement of performance in proposed technique such as facial wrinkles, which can be eliminated with the support of photographer.

J. Lu, V.E. Liong, and J. Zhou [19] presented a Cost-Sensitive- Local Binary Feature Learning (CSLBFL) strategy for facial age estimation. In conventional facial age estimation methods, the hand-crafted or learned descriptors were implemented for feature representation. Here, CSLBFL scheme learns discriminative local features directly from raw pixels for face representation. Whereas, the facial age estimation is a cost-sensitive computer vision issue and local features are more robust to illumination and appearance variations than holistic features. In addition, the author proposed a cost-sensitive learning strategy in various measures for reducing the face patches. CS-LBFL technique were executed on four facial collections. So, the complexity of the age system is quiet high.

X. Luan, B. Fang, L. Liu, W. Yang, and J. Qian [20] presented a new method for face detection, named as Robust-Principal component analysis (RPCA) associated with the low-rank module. The related Sparse Error Component (SEC) demonstrates additional selecting data, which was considered as one of the advantages for face recognition. To signify the characteristics of the SEC, two descriptors were employed, namely smoothness and sparsity. The ratio based technique and recognition protocols were employed for categorizing the face images. Here, the consistency of stored data and maintaining the accuracy are the two major issues in this facial feature system, which leads to loss of information.

To overcome the above mentioned drawbacks, semantic feature descriptors with multi objective classification method is implemented that enhances the accuracy of overlapped age group classification or detection.

\section{Proposed methodology}

The overlapped age group estimation from facial image is divided into three major steps: image preprocessing, feature extraction and inter age group variations based on classification. A general block diagram of the proposed method is determined in the Fig. 1. 


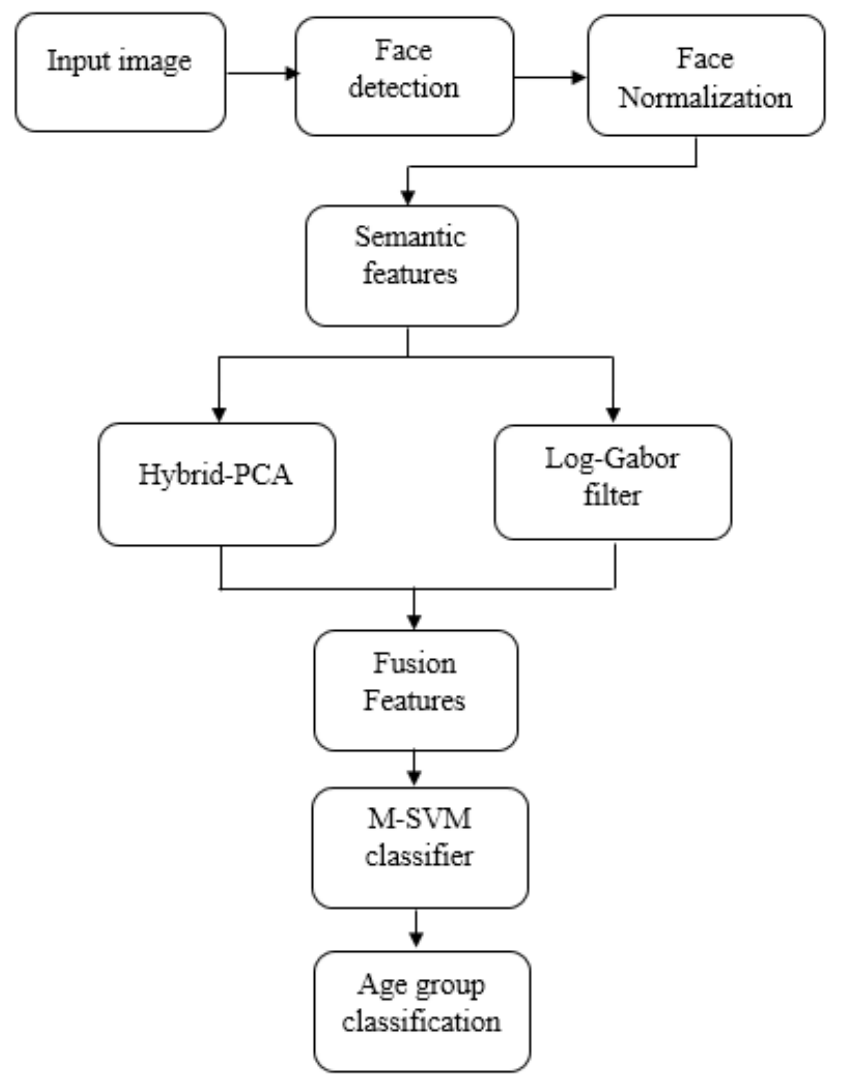

Figure.1 General block diagram of proposed method

\subsection{Pre-processing}

In pre-processing section, a normalization process is performed on the facial image for enhancing or de-noising the image. Normalization is a procedure that modifies the range of pixel intensity values for enhancing the quality of facial image by eliminating noise from the image and determine the alternations and deformation occurred in the facial image by inaccurate image capture. The sample normalized facial image is represented in the Fig.2.

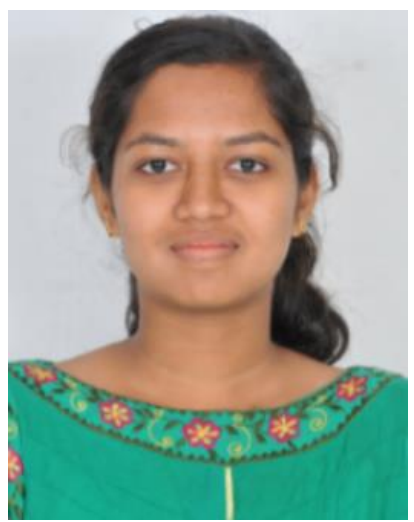

(a)

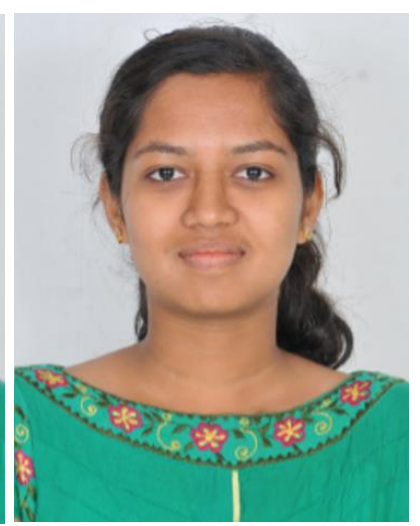

(b)
Figure.2 Sample normalized facial image: (a) Noisy image and (b) Normalized image
Image normalization consists of pixel intensity variations. The entire image is transformed into predefined values, because it is a pixel wise process. Suppose, the intensity range is $m$ to $n$, and the desire range is $p$ to $q$. In this condition, initial step in normalization methodology is subtracting $m$ from each pixel intensity. The general formula for image normalization methodology is determined in the Eq. (1).

$$
I N=(I-M i n)+\frac{\text { newMax-newMin }}{\text { Max-Min }}+\text { newMin }
$$

Where, $I$ is represented as original facial image, $I N$ is stated as new image, $(\operatorname{Min}=0, \operatorname{Max}=255)$ is specified as intensity range of original image and (newMin, newMax) is stated as intensity range of new image.

\subsection{Semantic Feature extraction}

In the proposed methodology, semantic feature extraction is performed (combination of HPCA and Log Gabor filter). Hence, HPCA extracts the hybrid appearance features by overlaying local and global appearance features of the face and Log Gabor filter 
extracts the texture appearance of the face. The fusion of dimension and textural features delivers an effective result in inter-age group class variation estimation.

\subsubsection{Hybrid Principal Component analysis}

Conventional, PCA is one of the statistical approaches often used to reduce the data dimension. Whereas, HPCA captures the hybrid appearance feature by superimposing local and global appearance features of the face. Generally, HPCA is classified into two types such as, extended PCA and extended sub-PCA. The quality of the prediction in HPCA is significant, because it mainly concentrates on covariance and variance structure of the new variables $x_{1}, x_{2} x_{3}, \ldots x_{p}$. These variable magnitudes are much higher than the other variables, hence they receive heavy weights. To avoid this reason, the variables are determined on scales with different ranges, otherwise the unit of the measurements are not equal. Let, $R$ be the sample correlation matrix calculated from $n$ observations on each principal component $p$ of random variables. The Eigen-value and Eigen-vector pairs of $R$ is represented as $\left(\varepsilon_{1}, e_{1}\right),\left(\varepsilon_{2}, e_{2}\right),\left(\varepsilon_{3}, e_{3}\right), \ldots . .\left(\varepsilon_{p}, e_{p}\right)$. The $i-t h$ sample principal component of a vector $x=$ $x_{1}, x_{2} x_{3}, \ldots x_{p}$ variable is given in the Eq. (2).

$$
e_{i} Z=e_{i 1} Z_{1}+e_{i 2} Z_{2}+\cdots e_{i p} Z_{p}, i=1,2,3, . . p
$$

Where, represents $i-t h$ $Z_{1}, Z_{2}, Z_{3}, \ldots . Z_{p}$ is the standardized vector observation.

In principal component, the sample variance is represented as $n_{i}$ and the sample covariance pairs are mentioned as zero. In addition, the total sample variance in all standardized variables is equal to the total sample variance in the principal component. Mathematically the standardized vector observation is expressed in the Eq. (3).

$$
Z_{k}=\frac{x_{k-} \bar{x}_{k}}{\sqrt{v_{k k}}}, \quad k=1,2,3, \ldots p
$$

Where, $x_{k}$ denotes sample mean and $v_{k k}$ is the sample variance of the variable $x_{k}$.

\subsubsection{Log Gabor filter}

Generally, Gabor filters are utilized to distinguish texture and also to detect and localize particular region in the image. Whereas, Gabor filters are quite time consuming, because several directions and orientations are used to handle the variability of image size. To overcome this drawback, log Gabor filter is proposed to construct the arbitrary bandwidth for optimizing the minimal spatial extent. Log Gabor filter in orientation domain is defined in polar coordinates, which is mentioned in the Eq. (4).

$$
H(f, \theta)=H_{f} \times H_{\theta}
$$

Where, $H_{f}$ is represented as the radial component and $H_{\theta}$ is sated as angle. The orientation factor is a Gaussian distance function according to the angle of polar coordinates, is mathematically expressed in the Eq. (5).

$$
H(f, \theta)=\exp \left\{\frac{-\left[\ln \left(\frac{f}{f_{0}}\right)\right]^{2}}{2\left[\ln \left(\frac{\sigma f}{f_{0}}\right)\right]^{2}}\right\} \times \exp \left\{\frac{-\left(\theta-\theta_{0}\right)^{2}}{2 \sigma_{\theta}^{2}}\right\}
$$

Where, $f_{0}$ is represented as central frequency, $\theta_{0}$ is stated as filter direction, $\sigma_{f}$ is defined as bandwidth. Log-Gabor filters are not really strict with directions and defining only two directions enables to handle facial images. Only two parameters remain to define, $f_{0}$ and $\sigma_{f}$ utilized to compute the radial bandwidth.

\subsection{Classification Method}

In classification section, semantic features are utilized to categorize the images by applying the classifier M-SVM. In this examination, several classes are needed to distinguish (overlapping agegroup classes). M-SVM classifier combines multiplebinary classes into a single objective function, and also perform significantly associated to other classifiers. A brief description about the M-SVM classifier is given below.

\subsubsection{Multi-Support Vector Machine}

Regular SVMs are basically a two-class classifier, to extend normal SVMs to multi classification, it is essential to modify the multi binary classification issues. Presently, for the Conventional-SVM (C$\mathrm{SVM})$, the multi-class classification is rehabilitated in two-class and for the $i-t h$ two-class issues, class $i$ is distinct from the residual classes.

Two of the prominent methodologies named as One-Against-One (1-a-1) and One-Against-All (1-aa). Here, the 1-a-a solution constructs a binary classifier for each class, which distinct the objects belongs to the class. In $n$ class, the 1-a-a method will generate a $n$ binary classifiers, and the $i-t h$ classifier is trained with the samples in $i-t h$ class 
with positive labels and all the remaining samples are trained with negative labels. The final outcome of the $n$ 1-a-a classifier correlates to the classifier with the maximum output value. Additionally, the 1-a-1 scheme is resultant from previous research on two class classifiers. The idea of this scheme is to generate all possible two class classifiers from a training set of $n$ classes, each classifier trained only two out of $n$ classes, and there would be $n \times(n-$ 1) $/ 2$ classifiers.

An effective way to diminish the multi-class issues is based on the decision function, which is constructed by assuming all classes. This technique is an extension to the SVM of facial image classification, to reduce multi-class issues in one step. It is represented in the Eqs. (6), (7) and (8).

$$
\min \phi(w, \varepsilon)=1 / 2 \sum_{m-1}^{k}\left(w_{m} \cdot w_{m}\right)+
$$

$c \sum_{i=1}^{l} \sum_{m \neq y i} \varepsilon_{i}^{m}$

Subjected to,

$$
\begin{array}{r}
\left(w_{y i} \cdot x i\right)+b_{y i} \geq\left(w_{y i} \cdot x i\right)+b_{m}+2-\varepsilon_{i}^{m},(7) \\
\varepsilon_{i}^{m} \geq 0, i=1,2, \ldots . . l, m, y i \epsilon\{1,2,3, \ldots . k\}, m \neq
\end{array}
$$

Where, $c$ is a user specified positive constant, $\varepsilon_{i}^{m}$ is mentioned as slack variables, $k$ is specified as the number of classes, $l$ is deliberated as training data point, each $y i$ is the class of the corresponding training data vectors $x i$.

Finally, the decision function is assumed in the Eq. (9)

$$
\begin{aligned}
& f(x)=\operatorname{argmax}\left[\left(w_{i} \cdot x\right)+b_{i}\right], \quad i= \\
& 1,2,3, \ldots . k
\end{aligned}
$$

Whereas, M-SVM shows a significant improvement over the overlapped age-group classification outcome and also the powerful degradation in error than correlated with other classification approaches.

\section{Result and discussion}

In this scenario, for experimental simulation, MATLAB (version 2017a) was employed on PC with $3.2 \mathrm{GHz}$ with i5 processor. The respective feature combinations were tested and trained by using $\mathrm{M}$ SVM on two different databases like EWC and MORPH. A brief description about the EWC and MORPH datasets are mentioned below.

\subsection{EWC dataset}

EWC of engineering is one of the prestigious educational institution in Bangalore. Among various disciplines, totally 69 individuals are undertaken for experimental analysis. The EWC female face database holds 29 individuals with 145 face images, each individual holds 5 facial images under altered pose variations and with different facial expressions. In EWC female facial database, the age variation starts from 16 to 47 . Likewise, the EWC male facial database holds 40 individuals with 200 face images, each individual holds 5 facial images under altered pose variations and with different facial expressions. In EWC male database, the age variation starts from 16 to 60 . The sample face images of EWC female and the male face database is mentioned in the Fig. 3.

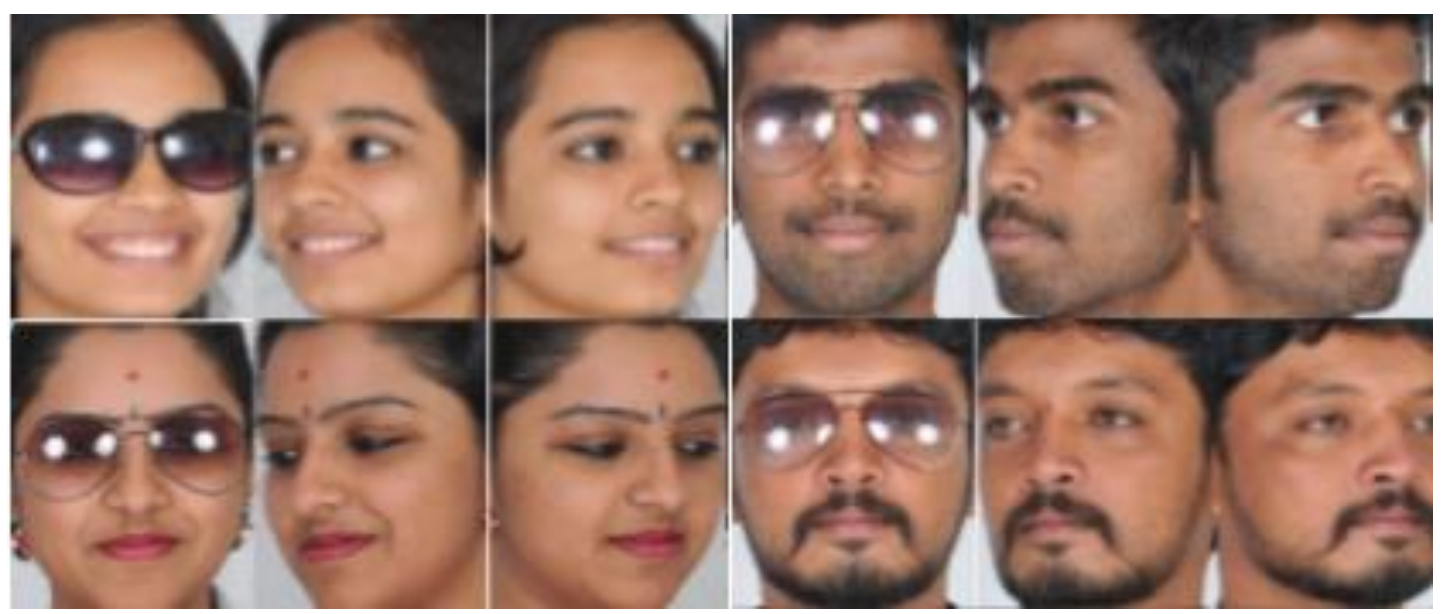

Figure.3 Sample EWC dataset image 


\subsubsection{Performance evaluation using EWC database}

The aging pattern is dissimilar for each person, individuals belonging to the same age group share some facial shape and texture similarities. In this section, male, female and the combination of both male and female facial images of EWC database is undertaken for experimental analysis. Totally, six different overlapped age group classes are assumed, which are described in the Table 1.

After separating the age group of EWC dataset, a semantic feature extraction is applied on the age group classes. By using the feature information, a multi-objective classifier (M-SVM) classifies the aging patterns. The predicted age of overlapped agegroup classes for semantic feature combination is determined, which is presented in the Table 2 and Fig. 4. In Table 2, the proposed semantic feature combination showed an improved result compared to the both person $\mathrm{A}$ and person $\mathrm{B}$. Person A represents male human prediction, and person $\mathrm{B}$ denotes female human prediction. By comparing the MAE value with semantic feature combination, the human observations show limited age prediction outcome, due to facial pose variation, facial expression, and image illuminance.

Table 1. Assumed age group classes

\begin{tabular}{|c|c|}
\hline Age-group classes & Age variation \\
\hline Class 1 & $15-25$ \\
\hline Class 2 & $20-30$ \\
\hline Class 3 & $26-35$ \\
\hline Class 4 & $31-40$ \\
\hline Class 5 & $36-45$ \\
\hline Class 6 & $41-50$ \\
\hline
\end{tabular}

Table 2. Predicted age for different age groups in EWC dataset

\begin{tabular}{|c|c|c|c|}
\hline $\begin{array}{c}\text { Age- } \\
\text { group } \\
\text { classes }\end{array}$ & $\begin{array}{c}\text { Proposed } \\
\text { semantic } \\
\text { feature } \\
\text { combination }\end{array}$ & $\begin{array}{c}\text { Person } \\
\text { A (Male) }\end{array}$ & $\begin{array}{c}\text { Person B } \\
\text { (Female) }\end{array}$ \\
\hline Class 1 & 4.56 & 5.42 & 4.89 \\
\hline Class 2 & 5.98 & 7.31 & 6.41 \\
\hline Class 3 & 3.63 & 4.87 & 8.23 \\
\hline Class 4 & 3.43 & 3.24 & 5.9 \\
\hline Class 5 & 6.10 & 6.45 & 7.2 \\
\hline Class 6 & 6.10 & 8.97 & 16.92 \\
\hline
\end{tabular}

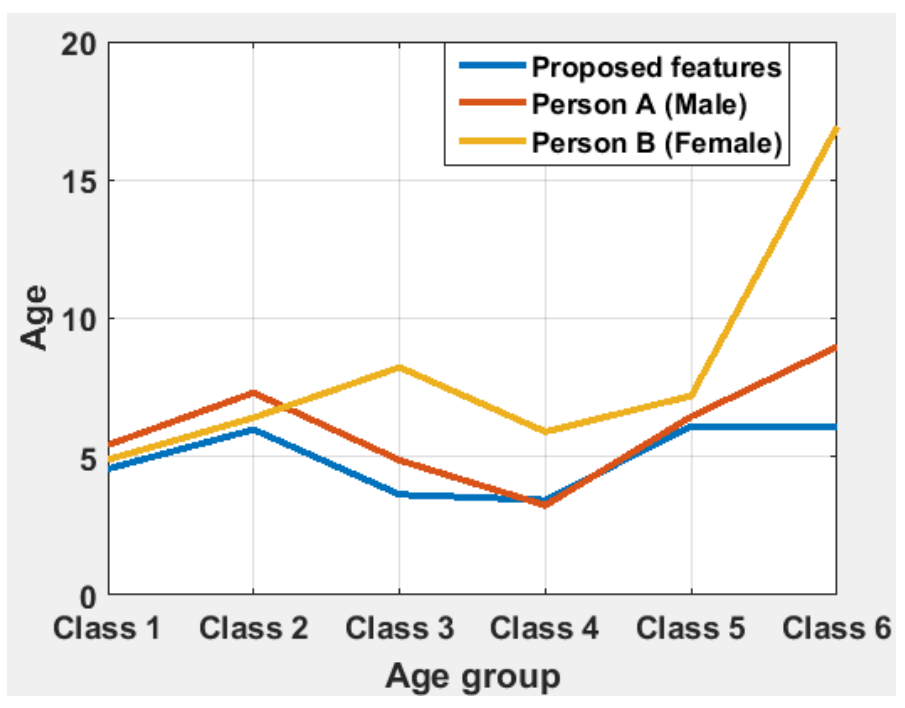

Figure.4 Comparison graph for EWC dataset

\subsection{MORPH dataset}

MORPH dataset comprises of 1724 facial images of 515 individuals. These images determine a diverse population with respect to age, gender, and ethnicity. There are 1278 images of African-American, and 436 images of Caucasian decent. In that, there are 294 images of females and 1430 images of males. The sample facial images of MORPH dataset are given in the Fig. 5. 


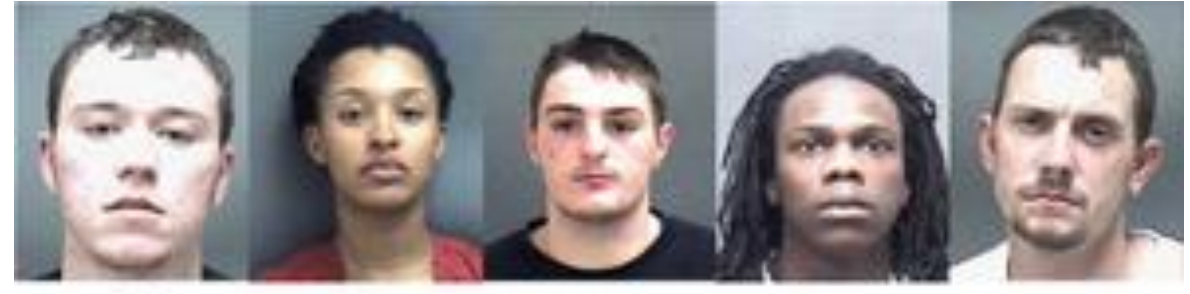

18

20

22

24

26
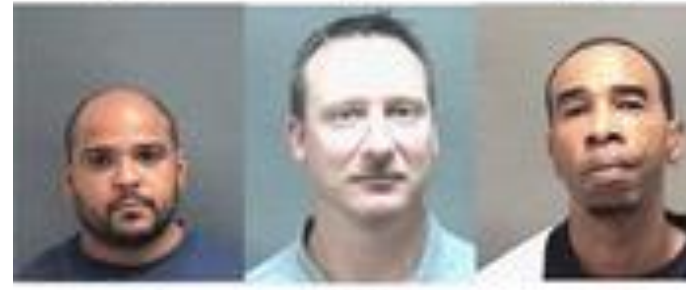

38

40

43

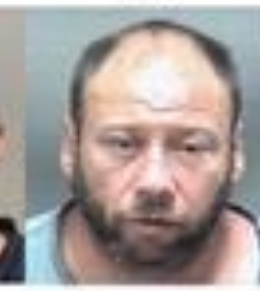

45

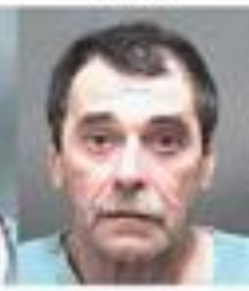

50

Figure.5 Sample MORPH dataset image

\subsubsection{Performance evaluation using MORPH database}

Different combination of male, female and the both male and female facial images of $\mathrm{MORPH}$ dataset is undertaken for overlapped age group analysis. In Table 3, different overlapped age-group classes of MORPH facial images are described. The predicted age of different age-group classes for MORPH dataset is determined, which is symbolized in the Fig. 6.
Table 3. Predicted age for different age groups in MORPH dataset

\begin{tabular}{|c|c|c|c|}
\hline $\begin{array}{c}\text { Age- } \\
\text { group } \\
\text { classes }\end{array}$ & $\begin{array}{l}\text { Proposed } \\
\text { semantic } \\
\text { feature } \\
\text { combination }\end{array}$ & $\begin{array}{c}\text { Person } \\
\text { A (Male) }\end{array}$ & $\begin{array}{c}\text { Person B } \\
\text { (Female) }\end{array}$ \\
\hline Class 1 & 3.23 & 7.44 & 9.78 \\
\hline Class 2 & 2.56 & 6.89 & 11.45 \\
\hline Class 3 & 4.13 & 8.08 & 8.06 \\
\hline Class 4 & 4.82 & 7.65 & 8.01 \\
\hline Class 5 & 2.90 & 8.0 & 9.56 \\
\hline Class 6 & 6.09 & 10.97 & 7.02 \\
\hline
\end{tabular}

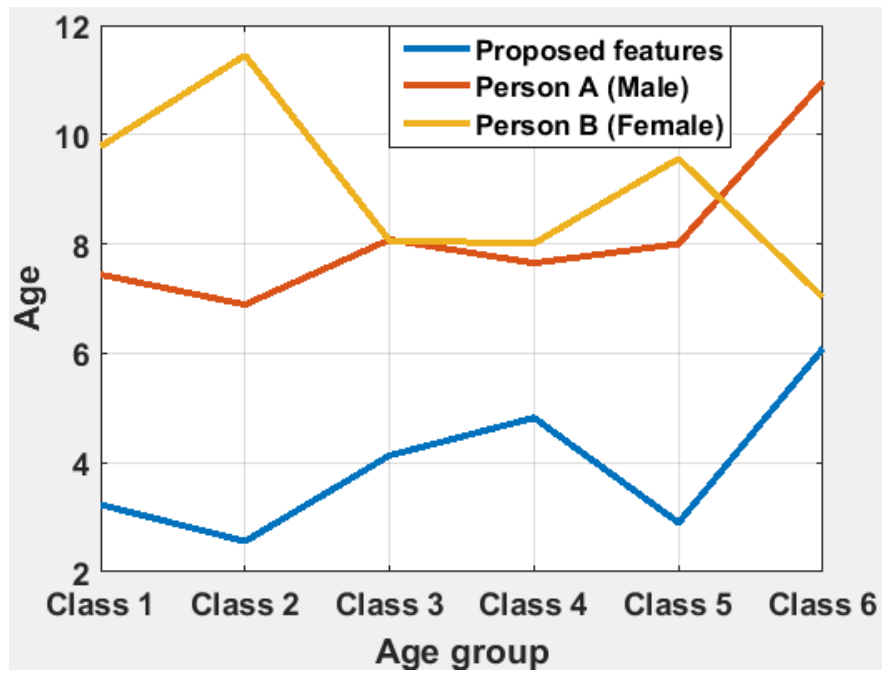

Figure.6 Comparison graph for MORPH dataset 
Table 4. Comparison of proposed outcome with existing work

\begin{tabular}{|c|c|c|c|c|c|c|c|}
\hline Dataset & Methods & Class 1 & Class 2 & Class 3 & Class 4 & Class 5 & Class 6 \\
\hline & LGBP [21] & $\mathbf{7 . 4 5}$ & $\mathbf{6 . 7 3}$ & $\mathbf{5 . 8}$ & $\mathbf{8 . 9}$ & $\mathbf{7 . 3 4}$ & $\mathbf{6 . 9 7}$ \\
\cline { 2 - 8 } EWC & Proposed & 4.56 & 5.98 & 3.63 & 3.43 & 6.10 & 6.89 \\
\hline \multirow{3}{*}{ MORPH } & LGBP [21] & 9.13 & 6.5 & 5.34 & 7.06 & 5.23 & 5.43 \\
\cline { 2 - 8 } & Proposed & $\mathbf{3 . 2 3}$ & $\mathbf{2 . 5 6}$ & $\mathbf{4 . 1 3}$ & $\mathbf{4 . 8 2}$ & $\mathbf{2 . 9 0}$ & $\mathbf{6 . 0 9}$ \\
\hline \multirow{3}{*}{ FG-NET } & SKNN-RKNN [22] & 1.59 & 2.48 & 2.50 & 3.68 & 15.9 & 17.78 \\
\cline { 2 - 8 } & Proposed & $\mathbf{2 . 6 7}$ & $\mathbf{3 . 2}$ & $\mathbf{3 . 2 3}$ & $\mathbf{3 . 7 8}$ & $\mathbf{4 . 8 9}$ & $\mathbf{5 . 1 2}$ \\
\hline
\end{tabular}

The comparative results of the age group analysis for different kinds of dataset is shown in the Table 4. Here, our proposed method performs significantly in overlapping age groups compared to the existing work [21], [22]. M. Kilinc and Y.S. Akgul, [21] presented an age estimation system that utilized an effective feature descriptor methodology Local Gabor Binary Patterns (LGBP), which was the combination of geometric and textural features. Then the probabilities of a face image belong to each overlapping age groups were determined by a group of classifiers. Then an interpolation based method was utilized to deliver the final estimated age.

Y. Liang [22] proposed two efficient classifiers, Sequence K-Nearest Neighbour (SKNN) and Ranking-KNN (R-KNN) for predicting age group and value. Especially, R-KNN uses the ordinal information between samples in estimation procedure rather than samples as separate individuals. Compared to these existing schemes, our proposed method works effectively in terms of MAE value.

\section{Conclusion}

This paper proposed a semantic feature extraction approach, and multi objective classification for facial overlapped age group estimation. The proposed approach showed an effective outcome on factors that affect the overlapped age estimation such as, lighting, illuminance, pose variation, occlusion with light, etc. In this experiment, we used the combination of geometric features and textural feature set (HPCA, and $\log$ Gabor filter). Employment of the textural method in geometric features improved the classification rates considerably. The combination of HPCA, and log Gabor filter in the M-SVM algorithm showed 5.098, 3.955 and 3.815, average MAE of age estimation for EWC, MORPH and FGNET databases, respectively. In the future work, we mainly concentrate on automatic age class generating scheme based on the characteristics of the dataset. At the same time, we try to improve the proposed age group estimation algorithm, since its result has a crucial effect on the entire system's performance.

\section{References}

[1] R. Sharma, and M.S. Patterh, "Indian face age database: A database for face recognition with age variation", International Journal of Computer Applications, Vol.126, No.5, pp.21-27, 2015.

[2] S. Ubaid, S. Das, and M.P. Imthiyas, "Human age prediction and classification using facial image", International Journal on Computer Science and Engineering, Vol.5, No.5, pp.357-361, 2013.

[3] D. Bhat, and V.K. Patil, "Human Age Estimation Based on Facial Aging Patterns", International Research Journal of Engineering and Technology (IRJET), Vol.3, No.7, pp.124-128, 2016.

[4] H. Han, C. Otto, A.K. Jain, "Age estimation from face images: Human vs. machine performance", In: Proc. of International Conf. On Biometrics (ICB), Madrid, Spain, pp.1-8, 2013.

[5] C.T. Lin, D.L. Li, J.H. Lai, M.F. Han, and J.Y. Chang, "Automatic Age Estimation System for Face Images", International Journal of Advanced Robotic Systems, Vol. 9, No.5, pp.1-9. 2012.

[6] P.K. Sai, J.G. Wang, and E.K. Teoh, "Facial age range estimation with extreme learning machines", Neurocomputing, Vol.149, pp.364372, 2015.

[7] M.Y. El Dib, and H.M. Onsi, "Human age estimation framework using different facial parts", Egyptian Informatics Journal, Vol.12, No.1, pp.53-59, 2011.

[8] X. Geng, C. Yin, and Z.H. Zhou, "Facial age estimation by learning from label distributions", IEEE Transactions on Pattern Analysis and Machine Intelligence, Vol.35, No.10, pp.24012412, 2013.

[9] H.B. Ali, and D.M. Powers, "Fusion based fast ICA method: Facial expression recognition", Journal of Image and Graphics, Vol.2, No.1, pp.1-7, 2014.

[10] J. Kou, J.X. Du, and C.M. Zhai, "Integration of global and local feature for age estimation of facial images", In: Proc. of International Conf. 
On Intelligent Computing, Berlin Heidelberg, pp. 455-462, 2012.

[11] S. Kumar, S. Ranjitha, and H. N. Suresh, "An Active Age Estimation of Facial image using Anthropometric Model and Fast ICA", Journal of Engineering Science \& Technology Review, Vol.10, No.1, pp.100-106, 2017.

[12] S.N. Kohail, "Using artificial neural network for human age estimation based on facial images", In: Proc. of International Conf. On Innovations in Information Technology (IIT), pp. 215-219, 2012.

[13] P.A. Krishna, A. Satish, V.G. Jagan Mohan, B. Dinesh, and A. Shaik, "Real Time Face Tracking by Using Naïve Bayes Classifier", International Journal of Innovative Research in Computer and Communication Engineering, Vol.2, No.1, pp.2692-2698, 2014.

[14] Z. Qawaqneh, A.A. Mallouh, and B.D Barkana, "Age and gender classification from speech and face images by jointly fine-tuned deep neural networks", Expert Systems with Applications, Vol.85, pp.76-86, 2017

[15] B. Julianson, L. Kim, M. Arby, S. Mehdi, and M. Chuchi, "Age Estimation Using Support Vector Machine-Sequential Minimal Optimization", Journal of Image and Graphics, Vol.2, No.2, pp.145-150, 2014.

[16] A. Günay, and V.V. Nabiyev, "Age Estimation Based on Hybrid Features of Facial Images", In: Proc. of International Conf. On Information Sciences and Systems, pp.295-304, 2015.

[17] T. Wu, P. Turaga, and R. Chellappa, "Age estimation and face verification across aging using landmarks", IEEE Transactions on Information Forensics and Security, Vol.7, No.6, pp.1780-1788, 2012.

[18] J.K. Pontes, A.S. Britto, C. Fookes, and A.L. Koerich, "A flexible hierarchical approach for facial age estimation based on multiple features", Pattern Recognition, Vol.54, pp.34-51, 2016.

[19] J. Lu, V.E. Liong, and J. Zhou, "Cost-sensitive local binary feature learning for facial age estimation", IEEE Transactions on Image Processing, Vol.24, No.12, pp.5356-5368, 2015.

[20] X. Luan, B. Fang, L. Liu, W. Yang, and J. Qian, "Extracting sparse error of robust PCA for face recognition in the presence of varying illumination and occlusion", Pattern Recognition, Vol.47, No.2, pp.495-508, 2014.

[21] M. Kilinc, and Y.S. Akgul, "Automatic human age estimation using overlapped age groups", In: Proc. of International Conf. On Computer Vision, Imaging and Computer Graphics, Berlin, Heidelberg, pp.313-325, 2013.
[22] Y. Liang, X. Wang, L. Zhang, and Z. Wang, "A hierarchical framework for facial age estimation", Mathematical Problems in Engineering, 2014. 